\title{
THE COMPLEXITY OF A MODULE AND ELEMENTARY ABELIAN SUBGROUPS: A GEOMETRIC APPROACH
}

\author{
PETER SYMONDS
}

(Communicated by Warren J. Wong)

\begin{abstract}
We present a proof of the theorem of Alperin and Evens that the complexity of a module is determined by the complexities of its restrictions to elementary abelian subgroups. We use only well-known properties of the spectral sequence.
\end{abstract}

The foundational material for the complexity theory of a module over a finite group can all be proved by elementary methods except for the key result that the complexity is determined by the complexities of the restrictions to the elementary abelian subgroups [AE]. The usual proofs use a theorem of Serre [S] and involve careful investigation of the behavior of products with respect to the filtration of the Lyndon-Hochschild-Serre spectral sequence.

We shall actually prove the following:

Theorem [AE]. Let $G$ be a finite group, $k$ a field of characteristic $p$, and $M a$ $k G$-module. Then $\mathrm{cx}_{G}\left(H^{*}(G ; M)\right)=\max _{J}\left(\mathrm{cx}_{G}\left(H^{*}\left(J ; M \downarrow_{J}^{G}\right)\right)\right)$, where $J$ runs through the abelian p-subgroups of $G$ and $\mathrm{cx}_{G}$ denotes complexity.

Remark. The usual form of this theorem as in [AE] is stronger in that $J$ only runs through elementary abelian subgroups. However, the reduction from abelian to elementary abelian can be made as in [C], or by a simplified version of the proof in [AE]. I wish to thank the authors of [AE] for pointing out an error in the first version of this proof.

Proof. We may restrict to a Sylow $p$-subgroup of $G$ without changing the complexity. So we assume that $G$ is a $p$-group. Then $\operatorname{cx}_{G}(M)=\gamma\left(H^{*}(G ; M)\right)$, where $\gamma$ denotes the growth function. We now use induction on $|G|$. If $G$ is abelian then there is nothing to prove, so we may assume that $G$ has an irreducible complex representation, $V$ for example, of dimension $n \geq 2$. Let $\mathbb{P}(V)$ denote the projective space of $V$ and triangulate it in such a way that $G$ acts admissibly. The stabilizers of the cells are all proper subgroups.

Now consider the fibration:

$$
\mathbb{P}(V) \stackrel{i}{\rightarrow} E G \times_{G} \mathbb{P}(V) \rightarrow B G .
$$

Received by the editors March 27, 1990 and, in revised form, June 28, 1990.

1980 Mathematics Subject Classification (1985 Revision). Primary 20J06; Secondary 55T10. 
This leads to the spectral sequence for equivariant cohomology:

$$
E_{2}^{* *}=H^{*}\left(G ; H^{*}(\mathbb{P}(V) ; M)\right) \Rightarrow H_{G}^{*}(\mathbb{P}(V) ; M) .
$$

The action of $G$ on $H^{*}(\mathbb{P}(V) ; \mathbb{Z})$ is trivial, since it factors through an action of the unitary group, which is connected. Thus the $E_{2}$ page can be identified with $H^{*}(G ; M) \otimes H^{*}(\mathbb{P}(V) ; k)$. The canonical line bundle $l$ over $\mathbb{P}(V)$ extends to a line bundle $l^{\prime}$ over $E G \times{ }_{G} \mathbb{P}(V)$. As $l=i^{*} l^{\prime}$, we obtain $c_{1}(l)=i^{*} c_{1}\left(l^{\prime}\right)$, where $c_{1}$ denotes the first Chern class. Consider the diagram

$$
\begin{gathered}
H_{G}^{0}(\mathbb{P}(V) ; M) \otimes H_{G}^{*}(\mathbb{P}(V) ; k) \stackrel{i^{*}}{\rightarrow} M \otimes H^{*}(\mathbb{P}(V) ; k) \\
\downarrow \mathbb{R} \\
E_{\infty}^{*, 0}=H_{G}^{*}(\mathbb{P}(V) ; M) \stackrel{i^{*}}{\rightarrow} H^{*}(\mathbb{P}(V) ; M)=E_{2}^{*, 0} ;
\end{gathered}
$$

the upper horizontal arrow is surjective and therefore, so is the lower one. This forces the spectral sequence to collapse by the Leray-Hirsch Theorem [D, H], and so

$$
H_{G}^{s}(\mathbb{P}(V) ; M) \cong \bigoplus_{i=0}^{n} H^{s-2 i}(G ; M),
$$

(the nonzero terms are contained in a horizontal strip of width $2 n-1$ ). Hence $\gamma\left(H_{G}^{*}(\mathbb{P}(V) ; M)\right)=\gamma\left(H^{*}(G ; M)\right)$.

We can also consider the spectral sequence on cells [B, p. 173]:

$$
E_{1}^{p, q}=\bigoplus_{\substack{\sigma \in G \mathbb{P}(V) \\ \operatorname{dim} \sigma=p}} H^{q}\left(\operatorname{stab}_{G} \sigma ; M\right) \Rightarrow H_{G}^{p+q}(\mathbb{P}(V) ; M) .
$$

The succeeding pages of the spectral sequence can only have smaller groups as entries, so

$$
\operatorname{dim} H_{G}^{s}(\mathbb{P}(V) ; M) \leq \sum_{i=0}^{2(n-1)} \sum_{\operatorname{dim} \sigma=i} \operatorname{dim} H^{s-i}\left(\operatorname{stab}_{G} \sigma ; M\right),
$$

(the nonzero terms lie within a vertical strip.) Hence $\gamma\left(H_{G}^{*}(\mathbb{P}(V) ; M)\right) \leq$ $\max _{\sigma}\left(\gamma\left(H^{*}\left(\operatorname{stab}_{G} \sigma ; M\right)\right)\right)$.

All the stabilizers are proper subgroups and the inequality in the other direction is trivial, hence the proof is complete by induction.

\section{REFERENCES}

[AE] J. L. Alperin and L. Evens, Representations, resolutions and Quillen's dimension theorem, J. Pure Appl. Algebra 26 (1981), 1-9.

[B] K. S. Brown, Cohomology of groups, Springer-Verlag, New York, 1982.

[C] J. F. Carlson, Restrictions of modules over modular group algebras, J. Algebra 53 (1978), 334-343. 
[D] A. Dold, Relations between ordinary and extraordinary homology, Algebraic Topology, Student's Guide (J. F. Adams, ed.), London Math. Soc. Lecture Note Ser., No. 4, Cambridge Univ. Press, London and New York, 1972.

[H] D. Husemoller, Fibre bundles 2nd ed., Springer-Verlag, New York, 1975.

[S] J.-P. Serre, Sur la dimension cohomologique des groupes profinis, Topology 3 (1965), 413420.

Department of Mathematics, University of Alberta, Edmonton, Alberta T6G 2E1, CANADA 